\title{
Oscillator Strengths for Lines of Ionized Uranium (UII)
}

\author{
Charles H. Corliss \\ Institute for Basic Standards, National Bureau of Standards, Washington, D.C. 20234
}

(March 29, 1976)

\begin{abstract}
Oscillator strengths for 49 lines of U II recently measured by Voight can be used to calibrate the intensity scale of the U II lines in the NBS Tables of Spectral-Line Intensities and derive a larger set of oscillator strengths of lower precision but consistent with the new measurements. The standard deviation of the differences between the two sets of $g$-values for the 49 lines is 29 percent. Oscillator strengths of that precision are given for 776 additional lines from the NBS Intensity Tables. The uncertainty in absolute value is 67 percent.
\end{abstract}

Key words: Oscillator strengths for U II; transition probabilities for U II; uranium spectrum.

\section{Introduction}

Recently Voigt [1975] ${ }^{1}$ measured oscillator strengths for 49 lines of $\mathbf{U}_{\text {II }}$ in a wall-stabilized argon arc. His analysis of errors leads to the conclusion that, on a relative scale, his errors do not exceed 10 percent. In his table III of results for U II lines he compares his values with $g f$-values from Corliss and Bozman [1962]. The well-known energy dependent error of their values is clearly exhibited in this table. Nearly all the old values for lines originating from levels above $25000 \mathrm{~cm}^{-1}$ are larger than Voigt's values, while for lines originating from levels below $25000 \mathrm{~cm}^{-1}$ the old values are nearly all smaller. A new calibration of the level populations in the copper arc of Meggers, Corliss, and Scribner [1975] is clearly required.

\section{Comparison of Intensities with Oscillator Strengths}

We compare the intensities from Meggers, Corliss, and Scribner with the new oscillator strengths by the usual population plot of $\log I \lambda^{3} / g f_{v}$ versus upper energy level. The standard deviation of the residuals from the least-squares line fitted to the 49 points was 0.20 dex $( \pm 58 \%)$. Since Voigt's $g f$-values have a relative error of only 10 percent, we may interpret the residuals as mostly error in the intensities.

The errors in the intensities may be random, systematic or both. Random errors cannot be removed but systematic errors can be removed if they can be specified. There seem to be two possibilities of systematic error in the intensities, i.e., as a function of wavelength or of intensity. The residuals from the plot

${ }^{1}$ Years in brackets indicate the literature reference at the end of this paper. were plotted against both quantities and there was correlation in each case. It is not surprising that if one quantity were to show correlation the other would also, since atomic spectra usually show a correlation between wavelength and intensity. However, in this case the correlation of the residuals with intensity was better than with wavelength. This correlation plot and its least-squares fitted line are shown in figure 1 . The line can be represented by the equation $R=-0.894+0.323 \log I$. The only badly outlying point represents the spectrum line at $2941 \AA$.

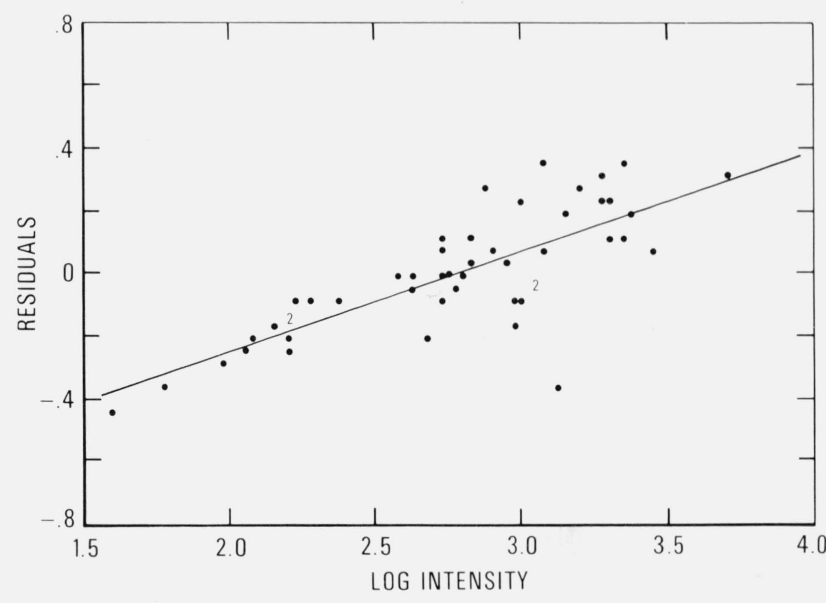

FIGURE 1. Residuals from a plot of $\log \mathrm{I}^{3} / \mathrm{gf}_{\mathrm{v}}$ versus energy level plotted versus $\log \mathrm{I}$

The intensity correlation shown above implies either that the intensity scale of Meggers, Corliss, and 


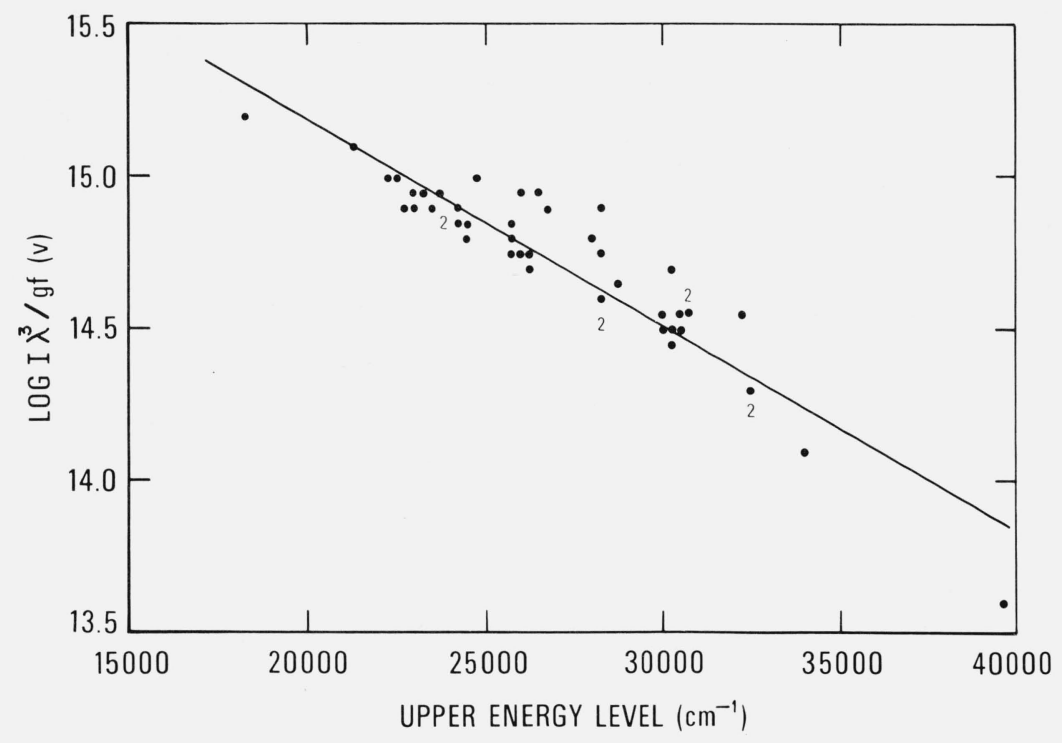

Figure 2. Population plot for the copper arc with the intensities adjusted according to the line in figure 1

Scribner is too expanded or that Voigt's scale is too compressed. ${ }^{2}$

\section{Derivation of New Values}

Figure 1 clearly demonstrates a systematic discrepancy between the intensity scale of $U$ II lines in Meggers, Corliss, and Scribner and Voigt's gf-values. To derive from our intensities $g f$-values consistent with Voigt's we should remove this systematic effect. By subtracting $R$ from $\log I$ we obtain a corrected intensity which has the systematic error removed. $\log I \lambda^{3} / g f_{v}$ is then recomputed using the corrected intensity and plotted in figure 2 . The standard deviation of the residuals is now reduced to 0.11 dex $( \pm 29 \%)$, which in fact represents also the standard deviation of the differences between Voigt's $g f$-values and $g f$-values derived from the corrected intensity scale of U II lines in the Tables of Spectral-Line Intensities.

The equation of the least-squares fitted line in figure 2 is $\log I \lambda^{3} / g f=16.531-0.00006717 E$. With this equation we calculate $g f$ for the 49 lines measured by Voigt and for 776 others.

\section{Results}

The results are given in the tables. In table 1 we give $\log g f$ as measured by Voigt and as calculated from the Tables of Spectral-Line Intensities. The differences for the 49 lines are given in the fourth column. The standard deviation of the differences is

\footnotetext{
${ }^{2}$ Bell and Upson [1971] investigated the intensity scale of Meggers, Corliss, and Scribner for the case of Fe I and concluded that the Fe I scale was too compressed. That conclusion does not support the present result.
}

TABLE 1. Comparison of log gf for lines of $\mathrm{U}$ II

\begin{tabular}{c|c|c|r}
\hline \hline $\begin{array}{c}\text { Wavelength } \\
\AA\end{array}$ & Log $g f(v)$ & $\log g f(c)$ & Difference \\
\hline 2941.92 & -0.18 & -0.47 & -0.29 \\
3111.62 & -0.72 & -0.86 & -0.14 \\
3305.90 & -0.92 & -0.99 & -0.07 \\
3550.82 & -1.28 & -1.01 & 0.27 \\
3670.07 & -0.59 & -0.72 & -0.13 \\
3700.58 & -1.34 & -1.21 & 0.13 \\
3701.52 & -0.62 & -0.69 & -0.07 \\
3746.41 & -1.00 & -0.84 & 0.16 \\
3748.68 & -0.64 & -0.73 & -0.09 \\
3782.84 & -1.06 & -0.89 & 0.17 \\
3826.51 & -1.39 & -1.17 & 0.22 \\
3831.46 & -0.70 & -0.59 & 0.11 \\
3854.66 & -0.59 & -0.54 & 0.05 \\
3859.58 & -0.62 & -0.62 & 0.00 \\
3865.92 & -0.85 & -0.77 & 0.08 \\
3881.46 & -1.00 & -0.80 & 0.20 \\
3890.36 & -1.00 & -0.86 & 0.14 \\
3932.03 & -0.82 & -0.89 & -0.07 \\
3985.80 & -0.72 & -0.71 & 0.01 \\
3992.54 & -1.13 & -1.02 & 0.11 \\
4017.72 & -0.96 & -0.91 & 0.05 \\
4050.04 & -1.11 & -0.99 & 0.12 \\
4051.91 & -0.92 & -0.95 & -0.03 \\
4062.55 & -1.12 & -1.17 & -0.05 \\
4067.76 & -1.03 & -0.90 & 0.13 \\
4090.14 & -0.70 & -0.78 & -0.08 \\
4116.10 & -1.19 & -1.19 & 0.00 \\
4124.73 & -1.26 & -1.28 & -0.02 \\
4128.34 & -1.15 & -1.10 & 0.05 \\
4141.23 & -0.70 & -0.80 & -0.10 \\
4171.59 & -0.96 & -0.92 & 0.04 \\
4241.67 & -0.68 & -0.83 & -0.15 \\
4244.37 & -1.28 & -1.33 & -0.05 \\
4341.69 & -1.21 & -1.24 & -0.03 \\
4372.57 & -1.54 & -1.63 & -0.09 \\
4373.41 & -1.62 & -1.68 & -0.06 \\
4472.34 & -1.18 & -1.28 & -0.10 \\
4515.28 & -1.54 & -1.56 & -0.02 \\
4538.19 & -1.55 & -1.53 & 0.02 \\
4543.63 & -1.16 & -1.24 & -0.08 \\
& & &
\end{tabular}


TABLE 1. Comparison of log of for lines of $\mathrm{U} \mathrm{II}-$ Continued

\begin{tabular}{c|c|c|c}
\hline \hline $\begin{array}{c}\text { Wavelength } \\
\text { A }\end{array}$ & $\log g f(v)$ & $\log g f(c)$ & Difference \\
\hline 4567.69 & -1.64 & -1.73 & -0.09 \\
4569.91 & -1.68 & -1.72 & -0.04 \\
4570.99 & -1.64 & -1.68 & -0.04 \\
4573.69 & -1.48 & -1.54 & -0.06 \\
4641.65 & -1.43 & -1.42 & 0.01 \\
4689.07 & -1.68 & -1.68 & 0.00 \\
4722.73 & -1.55 & -1.58 & -0.03 \\
4731.60 & -1.48 & -1.48 & 0.00 \\
5492.97 & -1.60 & -1.70 & -0.10 \\
\hline
\end{tabular}

0.11 dex $( \pm 29 \%)$. In table 2 we list the wavelength in Angstroms, energy levels in $\mathrm{cm}^{-1}$, the weighted transition probability $g A$ in units of $10^{8}$ transitions per second, the weighted oscillator strength, and $\log g f$ for 825 lines of U II from Meggers, Corliss, and Scribner. Classifications for 680 of these lines are given in the Intensity Tables and 145 more were classified with new levels kindly sent to us by Jean Blaise and Leon Radziemski from the Los Alamos Scientific
Laboratory. Earlier references for levels are given in the Intensity Tables.

In a number of cases the intensities in Meggers, Corliss, and Scribner represent the summation of unresolved pairs of lines. When both of the lines originate from the ion, we divided the intensity according to the ratio given in Steinhaus et al. [1972]. The remaining unresolved pairs were not used in this paper.

The error in the relative scale of $g f$ as determined above is about 30 percent. To calculate the error in the absolute scale, we add (quadratically) the absolute error of 60 percent determined by Voigt for his absolute scale with which we are calibrated. The uncertainty in our absolute scale is thus about 67 percent. This large absolute error arises from the uncertainty in the continuous background which had to be subtracted from a faint U I line during Voigt's measurement of the relative intensity of a $U_{I}$ and a U II line in his wall-stabilized arc. A direct measurement of a lifetime in U II would avoid this source of error.

TABLE 2. Oscillator strengths for lines of U II

\begin{tabular}{|c|c|c|c|c|c|c|c|c|c|}
\hline $\begin{array}{c}\text { Wavelength } \\
\AA\end{array}$ & $\begin{array}{c}\text { Energy levels } \\
\mathrm{cm}^{-1}\end{array}$ & $\begin{array}{c}\mathrm{gA} \\
10^{8 /} \mathrm{s} \\
\end{array}$ & gf & $\log g f$ & $\begin{array}{c}\text { Wavelength } \\
\AA \\
\end{array}$ & $\begin{array}{c}\text { Energy levels } \\
\mathrm{cm}^{-1}\end{array}$ & $\begin{array}{c}\mathrm{gA} \\
10^{8 / \mathrm{S}}\end{array}$ & gf & $\log g f$ \\
\hline 2419.57 & $0-41317$ & .50 & .044 & -1.36 & 2778.45 & $9553-45533$ & .91 & .11 & -.98 \\
\hline 2498.83 & $5526-45533$ & .41 & .038 & -1.42 & 2780.04 & $0-35960$ & .39 & .045 & -1.35 \\
\hline 2514.77 & $4420-44173$ & .72 & .069 & -1.16 & 2784.67 & $289-36189$ & .37 & .043 & -1.36 \\
\hline 2549.30 & $4420-43635$ & .68 & .066 & -1.18 & 2784.92 & $8276-44173$ & .74 & .086 & -1.07 \\
\hline 2556.19 & $0-39108$ & .74 & .072 & -1.14 & 2793.94 & $289-36070$ & 1.1 & .13 & -.90 \\
\hline 2565.41 & 0-38968 & 1.0 & .099 & -1.00 & 2797.30 & $0-35738$ & .22 & .026 & -1.59 \\
\hline 2568.98 & $5259-44173$ & .37 & .036 & -1.44 & 2802.56 & $289-35960$ & 1.1 & .13 & -.89 \\
\hline 2569.71 & $0-38903$ & .84 & .083 & -1.08 & 2805.24 & $914-36551$ & .40 & .047 & -1.33 \\
\hline 2584.42 & $0-38681$ & .42 & .043 & -1.37 & 2810.35 & $2294-37867$ & .39 & .046 & -1.34 \\
\hline 2616.07 & $0-38213$ & .33 & .034 & -1.46 & 2811.38 & $1749-37308$ & .64 & .076 & -1.12 \\
\hline 2621.81 & $0-38130$ & .25 & .025 & -1.59 & 2813.04 & $4706-40244$ & .71 & .084 & -1.08 \\
\hline 2639.84 & $0-37869$ & .38 & .040 & -1.40 & 2815.98 & $289-35790$ & .35 & .042 & -1.38 \\
\hline 2641.55 & $5790-43635$ & .58 & .061 & -1.21 & 2821.12 & $914-36351$ & 1.2 & .14 & -.86 \\
\hline 2644.12 & $0-37808$ & .38 & .040 & -1.40 & 2824.37 & $289-35684$ & .61 & .073 & -1.13 \\
\hline 2645.47 & 0-37789 & .90 & .095 & -1.02 & 2832.06 & $5259-40559$ & 2.3 & .28 & -.55 \\
\hline 2647.02 & $914-38681$ & .18 & .019 & -1.73 & 2834.55 & 914-36183 & .26 & .031 & -1.51 \\
\hline 2659.02 & 0-37596 & .23 & .024 & -1.61 & 2839.89 & $289-35491$ & .67 & .081 & -1.09 \\
\hline 2660.14 & $289-37869$ & .39 & .041 & -1.39 & 2845.96 & $914-36042$ & .34 & .042 & -1.38 \\
\hline 2665.87 & $289-37789$ & .32 & .034 & -1.47 & 2846.14 & $8510-43635$ & .81 & .099 & -1.01 \\
\hline 2675.88 & $1749-39108$ & .47 & .050 & -1.30 & 2849.98 & $5259-40337$ & .49 & .060 & -1.23 \\
\hline 2676.41 & $6283-43635$ & 1.4 & .15 & -.84 & 2850.82 & $5526-40594$ & .51 & .062 & -1.21 \\
\hline 2683.28 & $8276-45533$ & 3.0 & .33 & -.48 & 2852.75 & $5526-40570$ & .81 & .099 & -1.00 \\
\hline 2685.98 & $1749-38968$ & .76 & .082 & -1.09 & 2853.57 & $1749-36782$ & .45 & .055 & -1.26 \\
\hline 2691.80 & $8394-45533$ & 1.1 & .12 & -.94 & 2854.92 & $1749-36766$ & .28 & .034 & -1.46 \\
\hline 2705.19 & $914-37869$ & .52 & .058 & -1.24 & 2860.47 & $0-34949$ & .55 & .068 & -1.17 \\
\hline 2709.51 & $4420-41317$ & .56 & .062 & -1.21 & 2862.62 & $4585-39508$ & .43 & .053 & -1.27 \\
\hline 2711.10 & 914-37789 & .24 & .027 & -1.57 & 2865.68 & $0-34885$ & 1.0 & .13 & -.90 \\
\hline 2715.54 & $2295-39108$ & .40 & .044 & -1.36 & 2869.37 & $1749-36589$ & .44 & .054 & -1.26 \\
\hline 2725.94 & $2294-38968$ & .47 & .052 & -1.28 & 2874.08 & $1749-36532$ & .48 & .060 & -1.22 \\
\hline 2739.39 & $289-36782$ & .40 & .045 & -1.34 & 2875.20 & $914-35684$ & .42 & .053 & -1.28 \\
\hline 2746.16 & $1749-38152$ & .42 & .047 & -1.33 & 2886.45 & $289-34923$ & .34 & .043 & -1.37 \\
\hline 2750.13 & $0-36351$ & .17 & .019 & -1.73 & 2887.59 & $9553-44173$ & 1.2 & .15 & -.82 \\
\hline 2754.16 & $914-37212$ & .86 & .098 & -1.01 & 2888.26 & $0-34612$ & .55 & .069 & -1.16 \\
\hline 2766.88 & $0-36131$ & .40 & .045 & -1.34 & 2889.63 & $289-34885$ & 1.2 & .15 & -.83 \\
\hline 2776.29 & $4585-40594$ & .46 & .053 & -1.27 & 2894.14 & $5259-39801$ & 1.0 & .13 & -.88 \\
\hline
\end{tabular}


TABLE 2. Oscillator strengths for lines of $\mathrm{U}$ II-Continued

\begin{tabular}{|c|c|c|c|c|c|c|c|c|c|}
\hline $\begin{array}{c}\text { Wavelength } \\
\AA\end{array}$ & $\begin{array}{c}\text { Energy levels } \\
\mathrm{cm}^{-1}\end{array}$ & $\begin{array}{c}\mathrm{gA} \\
10^{8 / \mathrm{s}} \mathrm{s}\end{array}$ & gf & $\log g f$ & $\begin{array}{c}\text { Wavelength } \\
\AA\end{array}$ & $\begin{array}{c}\text { Energy levels } \\
\mathrm{cm}^{-1}\end{array}$ & $\begin{array}{c}\text { gA } \\
10^{8 /} / \mathrm{s}\end{array}$ & gf & $\log g f$ \\
\hline 2898.13 & $5259-39754$ & .62 & .078 & -1.11 & 3046.46 & $289-33104$ & .27 & .038 & -1.42 \\
\hline 2898.71 & $2294-36782$ & .39 & .049 & -1.31 & 3050.20 & $0-32775$ & .55 & .077 & -1.11 \\
\hline 2902.81 & $0-34439$ & .20 & .025 & -1.60 & 3056.72 & $289-32994$ & .27 & .038 & -1.42 \\
\hline 2904.51 & $289-34708$ & .39 & .049 & -1.31 & 3062.54 & $1749-34392$ & .75 & .11 & -.97 \\
\hline 2912.58 & $289-34612$ & .28 & .036 & -1.45 & 3063.88 & $2295-34923$ & .23 & .032 & -1.49 \\
\hline 2914.25 & $289-34593$ & .47 & .060 & -1.22 & 3066.87 & $8755-41352$ & .72 & .10 & -.99 \\
\hline 2918.97 & $5260-39508$ & .65 & .083 & -1.08 & 3068.65 & $5790-38368$ & .79 & .11 & -.95 \\
\hline 2923.17 & $0-34199$ & .19 & .025 & -1.60 & 3075.45 & $289-32795$ & .19 & .027 & -1.56 \\
\hline 2925.98 & $1749-35915$ & .38 & .048 & -1.32 & 3081.19 & $8510-40956$ & .86 & .12 & -.91 \\
\hline 2927.38 & $289-34439$ & .46 & .059 & -1.23 & 3084.24 & $2295-34708$ & .26 & .037 & -1.43 \\
\hline 2930.59 & $0-34112$ & .22 & .029 & -1.54 & 3086.70 & $5401-37789$ & .36 & .051 & -1.29 \\
\hline 2931.89 & $0-34097$ & 34 & .044 & -1.36 & 3086.80 & $11787-44173$ & .96 & .14 & -.86 \\
\hline 2936.45 & $2294-36339$ & .55 & .071 & -1.15 & 3087.11 & $2294-34678$ & .26 & .037 & -1.43 \\
\hline 2936.78 & $1749-35790$ & .25 & .032 & -1.49 & 3088.99 & $1749-34112$ & .38 & .054 & -1.27 \\
\hline 2937.35 & $914-34949$ & .26 & .033 & -1.48 & 3090.36 & $289-32638$ & .26 & .037 & -1.43 \\
\hline 2939.49 & $9626-43635$ & .98 & .13 & -.89 & 3094.83 & $289-32591$ & .19 & .027 & -1.57 \\
\hline 2941.92 & $5527-39508$ & 2.6 & .34 & -.47 & 3095.04 & $1749-34049$ & .43 & .061 & -1.21 \\
\hline 2942.12 & $2294-36273$ & .48 & .062 & -1.21 & 3095.23 & $2295-34593$ & .35 & .050 & -1.30 \\
\hline 2942.85 & $915-34885$ & .44 & .057 & -1.24 & 3095.75 & $5259-37552$ & .79 & .11 & -.95 \\
\hline 2943.40 & $4420-38385$ & .44 & .057 & -1.25 & 3098.01 & $5716-37985$ & .84 & .12 & -.92 \\
\hline 2945.89 & $1749-35684$ & .43 & .057 & -1.25 & 3103.77 & $914-33124$ & .20 & .030 & -1.53 \\
\hline 2948.09 & $289-34199$ & .46 & .060 & -1.22 & 3105.10 & $2294-34490$ & .29 & .042 & -1.37 \\
\hline 2949.61 & $4420-38313$ & .65 & .085 & -1.07 & 310565 & $5526-37716$ & 42 & .060 & -1.22 \\
\hline 2955.65 & $289-34112$ & .36 & .048 & -1.32 & $\begin{array}{l}5100.00 \\
311162\end{array}$ & $1749-33877$ & 95 & 14 & -86 \\
\hline 2957.74 & $4585-38385$ & .56 & .073 & -1.14 & $\begin{array}{l}3111.02 \\
3112.25\end{array}$ & $289-32410$ & $\begin{array}{l}.93 \\
.29\end{array}$ & .043 & -1.37 \\
\hline 2959.85 & $2294-36070$ & .49 & .065 & -1.19 & 3119.35 & $5260-37308$ & 1.1 & .16 & -.81 \\
\hline 2962.78 & $1749-35491$ & .45 & .059 & -1.23 & 3121.09 & $4663-36694$ & .36 & .052 & -1.28 \\
\hline 2966.66 & $914-34612$ & .39 & .052 & -1.28 & 3121.33 & $0-32028$ & .20 & .029 & -1.53 \\
\hline 2967.94 & $289-33972$ & .65 & .086 & -1.07 & 3124.43 & $8347-40344$ & .86 & .13 & -.90 \\
\hline 2968.40 & 914-34593 & .31 & .041 & -1.39 & 3126.70 & $4663-36637$ & .45 & .066 & -1.18 \\
\hline 2970.48 & $6283-39938$ & .56 & .074 & -1.13 & 3129.73 & $7166-39108$ & .71 & .10 & -.98 \\
\hline 2976.35 & $289-33877$ & .51 & .067 & -1.17 & 3130.73 & $289-32221$ & .15 & .023 & -1.65 \\
\hline 2980.28 & $5259-38803$ & .47 & .063 & -1.20 & 3131.99 & $5716-37635$ & .57 & .083 & -1.08 \\
\hline 2982.74 & $289-33805$ & .42 & .057 & -1.25 & 3133,42 & $2295-34.199$ & .21 & .031 & -1.51 \\
\hline 2984.61 & $2295-35790$ & .81 & .11 & -.97 & 3133.92 & $1749-33648$ & .26 & .038 & -1.42 \\
\hline 2987.80 & $289-33748$ & .27 & .037 & -1.44 & 3136.89 & $6283-38152$ & .52 & .077 & -1.11 \\
\hline 2989.88 & $1749-35185$ & .49 & .066 & -1.18 & 3138.51 & $4420-36273$ & .34 & .050 & -1.30 \\
\hline 2999.22 & $2294-35627$ & .37 & .050 & -1.30 & 3139.60 & $5716-37558$ & 1.1 & .17 & -.78 \\
\hline 3000.09 & $4420-37743$ & .51 & .069 & -1.16 & 3141.95 & $2294-34112$ & .28 & .042 & -1.38 \\
\hline 3001.21 & $1749-35059$ & .27 & .036 & -1.44 & 3144.96 & $5716-37504$ & .94 & .14 & -.86 \\
\hline 3007.91 & $5667-38903$ & 1.0 & .14 & -86 & 3145.56 & $5527-37308$ & 1.0 & .15 & -.82 \\
\hline 3010.75 & $289-33493$ & .27 & .036 & -1.44 & 3146.75 & $4663-36433$ & .40 & .060 & -1.22 \\
\hline 3012.45 & $289-33475$ & .13 & .024 & -1.61 & 3148.56 & $0-31751$ & .20 & .029 & -1.54 \\
\hline 3012.71 & $914-34097$ & .29 & .040 & -1.40 & $\begin{array}{l}3151.08 \\
3150\end{array}$ & $1749-33475$ & .25 & .038 & -1.42 \\
\hline 3013.37 & $4420-37596$ & .63 & .086 & -1.06 & 3153.12 & $8853-40559$ & 1.8 & .27 & -.57 \\
\hline 3016.96 & $1749-34885$ & .47 & .065 & -1.19 & 3155.41 & $4663-36346$ & .40 & .059 & -1.23 \\
\hline 3018.10 & $5259-38383$ & .52 & .071 & -1.15 & 3155.86 & $2294-33972$ & .41 & .061 & -1.21 \\
\hline 3020.24 & $4663-37764$ & .41 & .056 & -1.25 & 3160.77 & $5716-37345$ & .55 & .082 & -1.09 \\
\hline 3022.21 & $1749-34827$ & .80 & .11 & -.96 & 3165.28 & $6283-37867$ & .37 & .056 & -1.25 \\
\hline 3031.99 & $4663-37635$ & 1.2 & .17 & -.77 & 3165.50 & $2294-33876$ & .35 & .053 & -1.28 \\
\hline 3033.19 & $1749-34708$ & .66 & .091 & -1.04 & 3170.86 & $5716-37244$ & .46 & .069 & -1.16 \\
\hline 3035.96 & $1749-34678$ & .25 & .035 & -1.45 & 3171.37 & $5260-36782$ & .43 & .065 & -1.19 \\
\hline 3038.05 & $0-32905$ & .26 & .037 & -1.44 & 3173.71 & $0-31499$ & .14 & .021 & -1.68 \\
\hline 3039.14 & $4663-37558$ & .54 & .075 & -1.12 & 3175.36 & $8853-40337$ & .74 & .11 & -.95 \\
\hline 3039.26 & $5259-38152$ & .70 & .096 & -1.02 & 3176.21 & $1749-33224$ & .43 & .065 & -1.19 \\
\hline 3040.46 & $914-33794$ & .22 & .031 & -1.51 & 3177.33 & $5716-37180$ & .79 & .12 & -.92 \\
\hline 3043.79 & $1749-34593$ & .34 & .048 & -1.32 & 3179.04 & $289-31736$ & .25 & .039 & -1.41 \\
\hline 3044.16 & $4663-37504$ & 1.0 & .14 & -.85 & 3179.83 & $914-32353$ & .28 & .042 & -1.37 \\
\hline
\end{tabular}


TABLE 2. Oscillator strengths for lines of U II-Continued

\begin{tabular}{|c|c|c|c|c|c|c|c|c|c|}
\hline $\begin{array}{c}\text { Wavelength } \\
\AA\end{array}$ & $\begin{array}{l}\text { Energy levels } \\
\qquad \mathrm{cm}^{-1}\end{array}$ & $\begin{array}{c}\mathrm{gA} \\
10^{8 /} \mathrm{s}\end{array}$ & $\mathrm{gf}$ & $\log g f$ & $\begin{array}{c}\text { Wavelength } \\
\AA\end{array}$ & $\begin{array}{c}\text { Energy levels } \\
\mathrm{cm}^{-1}\end{array}$ & $\begin{array}{c}\mathrm{gA} \\
10^{8 /} \mathrm{s}\end{array}$ & gf & $\log g f$ \\
\hline 3180.20 & $1749-33184$ & .32 & .048 & -1.32 & 3370.13 & $5260-34923$ & .29 & .050 & -1.30 \\
\hline 3185.71 & $1749-33130$ & .36 & .055 & -1.26 & 3372.01 & $289-29936$ & .20 & .035 & -1.46 \\
\hline 3188.34 & $1749-33104$ & .32 & .048 & -1.32 & 3375.78 & $8853-38468$ & .77 & .13 & -.88 \\
\hline 3190.89 & $5259-36589$ & .26 & .040 & -1.40 & 3376.55 & $2294-31902$ & .18 & .031 & -1.50 \\
\hline 3191.76 & $4663-35985$ & .24 & .036 & -1.44 & 3382.68 & $2294-31848$ & .13 & .023 & -1.64 \\
\hline 3200.14 & $5716-36956$ & .61 & .094 & -1.03 & 3384.45 & $289-29827$ & .20 & .035 & -1.46 \\
\hline 3206.05 & $914-32096$ & .36 & .056 & -1.25 & 3386.13 & $914-30438$ & .22 & .038 & -1.42 \\
\hline 3206.23 & $2295-33475$ & .26 & .040 & -1.40 & 3392.99 & $4585-34049$ & .19 & .032 & -1.49 \\
\hline 3213.09 & $8394-39508$ & .41 & .064 & -1.19 & 3393.91 & $2294-31751$ & .33 & .057 & -1.25 \\
\hline 3218.34 & $5526-36589$ & .55 & .085 & -1.07 & 3394.78 & $5260-34.708$ & .58 & .10 & -1.00 \\
\hline 3219.17 & $6283-37338$ & .47 & .074 & -1.13 & 3395.32 & $1749-31193$ & .30 & .052 & -1.28 \\
\hline 3224.26 & $5526-36532$ & .58 & .090 & -1.05 & 3398.26 & $5259-34678$ & .33 & .057 & -1.24 \\
\hline 3226.17 & $914-31902$ & .28 & .044 & -1.36 & 3401.01 & $289-29683$ & .24 & .041 & -1.38 \\
\hline 3232.16 & $289-31219$ & .51 & .080 & -1.09 & 3401.87 & $4585-33972$ & .19 & .032 & -1.49 \\
\hline 3235.23 & $8853-39754$ & .43 & .068 & -1.17 & 3403.55 & $5259-34632$ & .51 & .089 & -1.05 \\
\hline 3238.46 & $914-31784$ & .13 & .020 & -1.70 & 3406.27 & $915-30263$ & .22 & .038 & -1.42 \\
\hline 3240.35 & $8347-39199$ & .40 & .063 & -1.20 & 3411.53 & $2294-31598$ & .32 & .056 & -1.25 \\
\hline 3241.99 & $914-31751$ & .26 & .041 & -1.39 & 3412.10 & $8853-38152$ & .89 & .16 & -.81 \\
\hline 3244.79 & $2295-33104$ & .25 & .039 & -1.41 & 3421.69 & $8379-37596$ & .68 & .12 & -.92 \\
\hline 3246.39 & $289-31083$ & .24 & .037 & -1.43 & 3422.35 & $5401-34612$ & .21 & .036 & -1.44 \\
\hline 3253.75 & $289-31014$ & .18 & .029 & -1.54 & 3423.01 & $5526-34732$ & .44 & .077 & -1.12 \\
\hline 3257.26 & $8276-38968$ & .39 & .061 & -1.21 & 3424.56 & $1749-30941$ & .47 & .082 & -1.09 \\
\hline 3261.72 & $1749-32399$ & .35 & .056 & -1.25 & 3426.39 & $11382-40559$ & .90 & .16 & -.80 \\
\hline 3265.81 & $914-31526$ & .34 & .054 & -1.26 & 3430.48 & $6283-35425$ & .23 & .041 & -1.38 \\
\hline 3269.78 & $289-30863$ & .11 & .018 & -1.75 & 3431.54 & $5791-34923$ & .35 & .061 & -1.21 \\
\hline 3270.12 & $289-30860$ & .37 & .059 & -1.23 & 3433.71 & $289-29403$ & .093 & .016 & -1.79 \\
\hline 3271.45 & $5401-35960$ & .24 & .039 & -1.41 & 3434.15 & $1749-30860$ & .28 & .049 & -1.31 \\
\hline 3280.00 & $914-31393$ & .19 & .031 & -1.51 & 3436.78 & $4706-33794$ & .29 & .052 & -1.29 \\
\hline 3282.48 & 0-30455 & .17 & .027 & -1.57 & 3451.21 & $1749-30716$ & .18 & .033 & -1.49 \\
\hline 3283.10 & $914-31364$ & .22 & .036 & -1.44 & 3453.57 & $8853-37801$ & .99 & .18 & -.75 \\
\hline 3285.22 & $1749-32179$ & .34 & .055 & -1.26 & 3453.78 & $5667-34612$ & .33 & .060 & -1.22 \\
\hline 3288.21 & $289-30691$ & .36 & .058 & -1.24 & 3454.23 & $5790-34732$ & .57 & .10 & -.99 \\
\hline 3294.44 & $8521-38867$ & .53 & .086 & -1.07 & 3455.74 & $1749-30678$ & .25 & .045 & -1.35 \\
\hline 3299.70 & $2294-32591$ & .30 & .049 & -1.31 & 3457.05 & $5716-34634$ & .56 & .10 & -1.00 \\
\hline 3303.60 & $289-30550$ & .27 & .043 & -1.36 & 3457.71 & $5526-34439$ & .54 & .097 & -1.01 \\
\hline 3305.90 & $0-30240$ & .62 & .10 & -.99 & 3458.68 & $2294-31199$ & .12 & .022 & -1.66 \\
\hline 3307.55 & $5526-35751$ & .24 & .039 & -1.41 & 3472.51 & $11797-40586$ & 1.6 & .29 & -.54 \\
\hline 3311.67 & $1749-31936$ & .33 & .054 & -1.27 & 3472.56 & $2295-31083$ & .19 & .035 & -1.45 \\
\hline 3313.94 & $289-30455$ & .26 & .043 & -1.36 & 3474.99 & $914-29683$ & .16 & .028 & -1.55 \\
\hline 3318.79 & $4585-34708$ & .20 & .034 & -1.47 & 3476.44 & $8423-37180$ & .50 & .090 & -1.04 \\
\hline 3319.32 & $12549-42666$ & 1.1 & .18 & -.74 & 3477.50 & $4663-33411$ & .17 & .032 & -1.50 \\
\hline 3322.12 & $4585-34678$ & .42 & .069 & -1.16 & 3482.49 & $1749-30455$ & .49 & .089 & -1.05 \\
\hline 3325.66 & $0-30060$ & .16 & .026 & -1.58 & 3486.30 & $7598-36273$ & .37 & .068 & -1.17 \\
\hline 3327.50 & $914-30958$ & .18 & .030 & -1.52 & 3489.57 & $5791-34439$ & .42 & .078 & -1.11 \\
\hline 3329.92 & $2294-32316$ & .35 & .058 & -1.23 & 3490.24 & $914-29557$ & .24 & .044 & -1.36 \\
\hline 3332.42 & $5791-35790$ & .24 & .040 & -1.40 & 3493.33 & $5259-33877$ & .57 & .11 & -.98 \\
\hline 3336.68 & $5790-35751$ & .38 & .064 & -1.19 & 3494.84 & $10198-38803$ & 1.1 & .20 & -.71 \\
\hline 3337.79 & $289-30240$ & .31 & .052 & -1.28 & 3495.60 & $4585-33184$ & .17 & .031 & -1.51 \\
\hline 3338.48 & $915-30860$ & .18 & .030 & -1.52 & 3495.75 & $1749-30347$ & .11 & .020 & -1.70 \\
\hline 3341.66 & $2295-32211$ & .46 & .077 & -1.11 & 3496.42 & $1749-30341$ & .41 & .075 & -1.12 \\
\hline 3342.68 & $1749-31656$ & .32 & .053 & -1.27 & 3497.62 & $8755-37338$ & .51 & .094 & -1.03 \\
\hline 3344.87 & $5526-35414$ & .37 & .061 & -1.21 & 3499.33 & $2295-30863$ & .26 & .048 & -1.32 \\
\hline 3354.50 & $2294-32096$ & .33 & .055 & -1.26 & 3505.07 & $5259-33781$ & .50 & .091 & -1.04 \\
\hline 3355.11 & $289-30085$ & .10 & .017 & -1.77 & 3505.45 & $0-28518$ & .13 & .024 & -1.61 \\
\hline 3357.93 & $289-30060$ & .24 & .040 & -1.39 & 3508.85 & $1749-30240$ & .29 & .053 & -1.28 \\
\hline 3361.73 & $2294-32032$ & .22 & .037 & -1.43 & 3509.67 & $8853-37338$ & .99 & .18 & -.74 \\
\hline 3367.90 & $0-29683$ & .13 & .022 & -1.65 & 3511.58 & $289-28758$ & .14 & .025 & -1.60 \\
\hline 3368.81 & $6283-35958$ & .52 & .088 & -1.05 & 3513.37 & $8853-37308$ & .51 & .095 & -1.02 \\
\hline
\end{tabular}


TABLE 2. Oscillator strengths for lines of $\mathrm{U} \mathrm{II}-$ Continued

\begin{tabular}{|c|c|c|c|c|c|c|c|c|c|}
\hline $\begin{array}{c}\text { Wavelength } \\
\AA\end{array}$ & $\begin{array}{c}\begin{array}{c}\text { Energy levels } \\
\mathrm{cm}^{-1}\end{array} \\
\end{array}$ & $\begin{array}{r}\mathbf{g A} \\
10^{8 /} \mathrm{s} \\
\end{array}$ & gf & $\log g f$ & $\begin{array}{c}\text { Wavelength } \\
\AA\end{array}$ & $\begin{array}{c}\text { Energy levels } \\
\mathrm{cm}^{-1}\end{array}$ & $\begin{array}{c}\text { gA } \\
10^{8 /} \mathrm{s}\end{array}$ & gf & $\log g f$ \\
\hline 3515.24 & $44.20-32860$ & .22 & .041 & -1.39 & 3672.58 & $8853-36074$ & .52 & .11 & -.98 \\
\hline 3516.35 & $5667-34097$ & .31 & .058 & -1.24 & 3674.99 & $8755-35958$ & .23 & .048 & -1.32 \\
\hline 3517.05 & $6283-34708$ & .34 & .064 & -1.19 & 3675.08 & $5259-32462$ & .23 & .047 & -1.33 \\
\hline 3519.96 & $914-29316$ & .29 & .053 & -1.28 & 3676.56 & $6283-33475$ & .43 & .087 & -1.06 \\
\hline 3520.79 & $6283-34678$ & .40 & .075 & -1.13 & 3677.64 & $11784-38968$ & .82 & .17 & -.78 \\
\hline 3521.48 & $5259-33648$ & .25 & .047 & -1.33 & 3678.75 & $8347-35523$ & .77 & .16 & -.81 \\
\hline 3523.33 & $4663-33037$ & .17 & .031 & -1.51 & 3682.04 & $5259-32410$ & .34 & .069 & -1.16 \\
\hline 3523.57 & $11382-39754$ & 1.0 & .19 & -.72 & 3691.92 & $4706-31784$ & .55 & .11 & -.95 \\
\hline 3525.73 & $9241-37596$ & .34 & .063 & -1.20 & 3693.70 & $7547-34612$ & .61 & .12 & -.91 \\
\hline 3526.60 & $289-28636$ & .19 & .035 & -1.46 & 3697.93 & $8755-35790$ & .55 & .11 & -.94 \\
\hline 3528.69 & $10198-38529$ & .86 & .16 & -.80 & 3700.58 & $915-27929$ & .30 & .062 & -1.21 \\
\hline 3529.77 & $0-28322$ & .081 & .015 & -1.82 & 3701.52 & $5527-32535$ & 1.0 & .20 & -.69 \\
\hline 3531.11 & $1749-30060$ & .32 & .060 & -1.22 & 3705.04 & $2294-29277$ & .18 & .036 & -1.44 \\
\hline 3533.57 & $915-29206$ & .39 & .073 & -1.14 & 3705.97 & $10740-37716$ & .47 & .096 & -1.02 \\
\hline 3537.45 & $5259-33520$ & .29 & .054 & -1.27 & 3707.65 & $9626-36589$ & .39 & .081 & -1.09 \\
\hline 3540.46 & $5716-33953$ & .72 & .14 & -.87 & 3714.76 & $2295-29206$ & .21 & .043 & -1.37 \\
\hline 3543.16 & $5260-33475$ & .39 & .074 & -1.13 & 3717.42 & $8521-35414$ & .65 & .13 & -.87 \\
\hline 3544.21 & $6283-34490$ & .34 & .063 & -1.20 & 3718.11 & $1749-28636$ & .25 & .052 & -1.28 \\
\hline 3546.68 & $1749-29936$ & .23 & .043 & -1.37 & 3718.61 & $5526-32410$ & .27 & .055 & -1.26 \\
\hline 3547.19 & $1749-29932$ & .32 & .060 & -1.22 & 3724.98 & $1749-28587$ & .17 & .036 & -1.45 \\
\hline 3550.82 & $0-28154$ & .52 & .097 & -1.01 & 3725.07 & $5526-32364$ & .25 & .053 & -1.28 \\
\hline 3551.04 & $289-28441$ & .13 & .025 & -1.60 & 3725.65 & $7166-33999$ & .28 & .058 & -1.23 \\
\hline 3552.17 & $2294-30438$ & .30 & .057 & -1.25 & 3730.13 & $5790-32591$ & .23 & .047 & -1.33 \\
\hline 3560.44 & $1749-29827$ & .16 & .031 & -1.51 & 3731.67 & $5526-32316$ & .20 & .043 & -1.37 \\
\hline 3564.59 & $8347-36393$ & .62 & .12 & -.93 & 3732.62 & $914-27698$ & .22 & .046 & -1.34 \\
\hline 3564.88 & $9553-37596$ & .55 & .10 & -.98 & 3737.25 & $10740-37490$ & .75 & .16 & -.80 \\
\hline 3570.93 & $4420-32416$ & .15 & .029 & -1.53 & 3738.05 & $5791-32535$ & .67 & .14 & -.85 \\
\hline 3571.56 & $5790-33781$ & .26 & .050 & -1.30 & 3744.24 & $7598-34298$ & .55 & .12 & -.94 \\
\hline 3571.69 & $4420-32410$ & .25 & .047 & -1.33 & 3746.41 & $5527-32211$ & .69 & .15 & -.84 \\
\hline 3576.22 & $10198-38152$ & .82 & .16 & -.80 & 3747.14 & $8521-35201$ & .70 & .15 & -.83 \\
\hline 3578.72 & $8276-36211$ & 1.2 & .22 & -.65 & 3748.68 & $5716-32384$ & .89 & .19 & -.73 \\
\hline 3581.84 & $5716-33627$ & .54 & .10 & -.99 & 3752.66 & $5526-32166$ & .44 & .093 & -1.03 \\
\hline 3590.32 & $5260-33104$ & .43 & .083 & -1.08 & 3754.31 & $0-26628$ & .12 & .026 & -1.58 \\
\hline 3590.50 & $915-28758$ & .23 & .045 & -1.34 & 3755.48 & $5790-32410$ & .46 & .097 & -1.01 \\
\hline 3594.95 & $1749-29557$ & .25 & .048 & -1.32 & 3756.92 & $4706-31316$ & .30 & .063 & -1.20 \\
\hline 3596.88 & $1749-29543$ & .12 & .023 & -1.63 & 3759.23 & $5667-32261$ & .45 & .095 & -1.02 \\
\hline 3599.84 & $8347-36118$ & .44 & .085 & -1.07 & 3759.88 & $5259-31848$ & .20 & .043 & -1.37 \\
\hline 3605.48 & $1749-29476$ & .14 & .026 & -1.58 & 3760.88 & $0-26581$ & .14 & .031 & -1.52 \\
\hline 3606.32 & $4663-32384$ & .45 & .087 & -1.06 & 3761.96 & $914-27489$ & .13 & .027 & -1.57 \\
\hline 3608.96 & $8510-36211$ & .45 & .087 & -1.06 & 3762.11 & $1749-28322$ & .14 & .030 & -1.52 \\
\hline 3609.68 & $5716-33411$ & .40 & .078 & -1.11 & 3764.57 & $5716-32272$ & .56 & .12 & -.92 \\
\hline 3610.49 & $6283-33972$ & .32 & .062 & -1.21 & 3768.80 & $5790-32316$ & .35 & .075 & -1.13 \\
\hline 3611.24 & $2294-29978$ & .11 & .021 & -1.68 & 3772.82 & $8347-34845$ & .44 & .094 & -1.02 \\
\hline 3612.67 & $915-28587$ & .16 & .032 & -1.50 & 3780.72 & 915-27357 & .22 & .048 & -1.32 \\
\hline 3616.76 & $289-27930$ & .21 & .041 & -1.39 & 3782.84 & $289-26716$ & .60 & .13 & -.89 \\
\hline 3618.49 & $9553-37181$ & .52 & .10 & -.99 & 3783.84 & $5791-32211$ & .51 & .11 & -.96 \\
\hline 3623.06 & $915-28507$ & .26 & .051 & -1.29 & 3786.57 & $8521-34923$ & .33 & .071 & -1.15 \\
\hline 3628.35 & $8521-36074$ & .27 & .054 & -1.27 & 3787.23 & $5259-31656$ & .27 & .058 & -1.23 \\
\hline 3632.87 & $10198-37716$ & .35 & .070 & -1.15 & 3790.22 & $5790-32166$ & .22 & .046 & -1.33 \\
\hline 3635.40 & $0-27499$ & .12 & .023 & -1.64 & 3790.33 & $914-27290$ & .10 & .022 & -1.66 \\
\hline 3640.95 & $1749-29206$ & .21 & .042 & -1.38 & 3793.10 & $4585-30941$ & .51 & .11 & -.96 \\
\hline 3644.85 & $2294-29722$ & .078 & .016 & -1.81 & 3793.57 & $914-27267$ & .22 & .048 & -1.32 \\
\hline 3645.03 & 914-28341 & .22 & .043 & -1.36 & 3795.13 & $2294-28636$ & .19 & .041 & -1.39 \\
\hline 3649.51 & $4706-32099$ & .16 & .032 & -1.49 & 3796.54 & $4663-30996$ & .29 & .062 & -1.21 \\
\hline 3659.01 & $4706-32028$ & .26 & .052 & -1.29 & 3796.84 & $6445-32775$ & .38 & .082 & -1.09 \\
\hline 3662.33 & $4663-31961$ & .24 & .049 & -1.31 & 3799.20 & $8394-34708$ & .51 & .11 & -.96 \\
\hline 3666.21 & $8521-35790$ & .34 & .069 & -1.16 & 3802.27 & $2294-28587$ & .20 & .043 & -1.37 \\
\hline 3670.07 & $915-28154$ & .95 & .19 & -.72 & 3803.35 & $0-26285$ & .087 & .019 & -1.72 \\
\hline
\end{tabular}


TABLE 2. Oscillator strengths for lines of U II-Continued

\begin{tabular}{|c|c|c|c|c|c|c|c|c|c|}
\hline $\begin{array}{c}\text { Wavelength } \\
\AA\end{array}$ & $\begin{array}{c}\text { Energy levels } \\
\mathrm{cm}^{-1}\end{array}$ & $\begin{array}{r}\text { gA } \\
10^{8 /} \mathrm{s} \\
\end{array}$ & gf & $\log g f$ & $\begin{array}{c}\text { Wavelength } \\
\AA\end{array}$ & $\begin{array}{c}\text { Energy levels } \\
\mathrm{cm}^{-1}\end{array}$ & $\begin{array}{r}\text { gA } \\
10^{8 /} \mathrm{s} \\
\end{array}$ & gf & $\log g f$ \\
\hline 3809.22 & $5716-31961$ & .46 & .10 & -1.00 & 3927.76 & $6283-31736$ & .33 & .077 & -1.11 \\
\hline 3813.79 & $2295-28507$ & .27 & .059 & -1.23 & 3930.98 & $289-25720$ & .20 & .045 & -1.34 \\
\hline 3814.07 & $915-27126$ & .22 & .047 & -1.33 & 3932.03 & $289-25714$ & .55 & .13 & -.89 \\
\hline 3818.46 & $1749-27930$ & .11 & .025 & -1.61 & 3933.03 & $5259-30678$ & .18 & .041 & -1.39 \\
\hline 3822.54 & $4706-30859$ & .18 & .039 & -1.41 & 3935.38 & $2294-27698$ & .29 & .067 & -1.17 \\
\hline 3826.51 & $289-26415$ & .31 & .068 & -1.17 & 3940.49 & $914-26285$ & .18 & .042 & -1.38 \\
\hline 3829.03 & $915-27023$ & .16 & .035 & -1.46 & 3942.55 & $8755-34112$ & .41 & .096 & -1.02 \\
\hline 3829.39 & $4585-30691$ & .24 & .052 & -1.28 & 3944.13 & $4585-29932$ & .25 & .059 & -1.23 \\
\hline 3831.46 & $4663-30756$ & 1.2 & .26 & -.59 & 3953.58 & $2294-27581$ & .21 & .048 & -1.32 \\
\hline 3835.92 & $7598-33660$ & .39 & .086 & -1.07 & 3954.62 & $5716-30996$ & .25 & .058 & -1.24 \\
\hline 3838.15 & $2294-28341$ & .17 & .038 & -1.42 & 3954.67 & $8347-33627$ & .59 & .14 & -.86 \\
\hline 3845.12 & $5526-31526$ & .20 & .044 & -1.36 & 3962.77 & $4706-29934$ & .22 & .051 & -1.29 \\
\hline 3845.26 & $7547-33546$ & .37 & .082 & -1.09 & 3964.67 & $7166-32382$ & .37 & .088 & -1.06 \\
\hline 3845.37 & $8394-34392$ & .42 & .093 & -1.03 & 3964.96 & $0-25213$ & .093 & .022 & -1.66 \\
\hline 3848.60 & $5259-31235$ & .49 & .11 & -.96 & 3966.40 & $2295-27499$ & .18 & .041 & -1.38 \\
\hline 3849.71 & $8522-34490$ & .31 & .070 & -1.16 & 3966.52 & $289-25492$ & .24 & .056 & -1.25 \\
\hline 3849.85 & $0-25967$ & .12 & .026 & -1.58 & 3969.02 & $8400-33588$ & .28 & .066 & -1.18 \\
\hline 3854.66 & $4663-30599$ & 1.3 & .29 & -.54 & 3978.80 & $8394-33520$ & .38 & .090 & -1.04 \\
\hline 3859.58 & $289-26191$ & 1.1 & .24 & -.62 & 3985.80 & $5260-30341$ & .81 & .19 & -.71 \\
\hline 3864.30 & $4585-30455$ & .27 & .060 & -1.22 & 3988.64 & $8276-33340$ & .21 & .049 & -1.31 \\
\hline 3864.48 & $4663-30533$ & .27 & .061 & -1.21 & 3988.89 & $2295-27357$ & .15 & .035 & -1.46 \\
\hline 3865.92 & $2295-28154$ & .77 & .17 & -.77 & 3990.42 & $915-25967$ & .22 & .052 & -1.29 \\
\hline 3866.80 & $914-26768$ & .21 & .047 & -1.33 & 3992.54 & $5716-30756$ & .40 & .095 & -1.02 \\
\hline 3868.42 & $4421-30263$ & .16 & .037 & -1.43 & 3994.29 & $289-25317$ & .079 & .019 & -1.73 \\
\hline 3870.02 & $5667-31499$ & .32 & .071 & -1.15 & 4002.34 & $4706-29684$ & .15 & .037 & -1.43 \\
\hline 3871.88 & $8347-34167$ & .26 & .058 & -1.24 & 4003.20 & $2294-27267$ & .11 & .026 & -1.59 \\
\hline 3874.04 & $289-26094$ & .26 & .059 & -1.23 & 4004.06 & $1749-26716$ & .20 & .049 & -1.31 \\
\hline 3876.59 & $9626-35414$ & .36 & .082 & -1.09 & 4009.17 & $6283-31219$ & .20 & .047 & -1.32 \\
\hline 3878.09 & $8853-34632$ & .98 & .22 & -.66 & 4011.45 & $5401-30323$ & .16 & .039 & -1.41 \\
\hline 3881.46 & $4585-30341$ & .70 & .16 & -.80 & 4014.16 & $8755-33660$ & .22 & .053 & -1.28 \\
\hline 3882.36 & $1749-27499$ & .28 & .063 & -1.20 & 4017.72 & $5716-30599$ & .51 & .12 & -.91 \\
\hline 3883.28 & $8423-34167$ & .66 & .15 & -.83 & 4018.99 & $289-25163$ & .14 & .035 & -1.46 \\
\hline 3884.68 & $8755-34490$ & .51 & .11 & -.94 & 4026.02 & $2295-27126$ & .080 & .019 & -1.71 \\
\hline 3887.70 & $10198-35913$ & .52 & .12 & -.93 & 4031.31 & $914-25713$ & .10 & .025 & -1.60 \\
\hline 3890.36 & $289-25986$ & .61 & .14 & -.86 & 4033.43 & $4421-29206$ & .18 & .043 & -1.37 \\
\hline 3891.68 & $8510-34199$ & .30 & .069 & -1.16 & 4033.73 & $7598-32382$ & .32 & .079 & -1.10 \\
\hline 3892.41 & $8755-34439$ & .50 & .11 & -.94 & 4044.42 & $5259-29978$ & .44 & .11 & -.97 \\
\hline 3892.68 & $5260-30941$ & .56 & .13 & -.90 & 4050.04 & $0-24684$ & .42 & .10 & -.99 \\
\hline 3895.27 & $4421-30085$ & .23 & .052 & -1.29 & 4051.91 & $5260-29932$ & .45 & .11 & -.95 \\
\hline 3896.78 & $4585-30240$ & .43 & .097 & -1.01 & 4053.03 & $1749-26415$ & .12 & .031 & -1.51 \\
\hline 3897.05 & $10740-36393$ & .68 & .15 & -.81 & 4054.31 & $6283-30941$ & .35 & .087 & -1.06 \\
\hline 3897.69 & $5667-31316$ & .19 & .044 & -1.35 & 4058.19 & $289-24923$ & .18 & .044 & -1.36 \\
\hline 3899.07 & $4421-30060$ & .22 & .050 & -1.30 & 4060.10 & $4706-29329$ & .11 & .028 & -1.55 \\
\hline 3899.48 & $4663-30301$ & .27 & .061 & -1.22 & 4061.74 & $7598-32211$ & .22 & .054 & -1.27 \\
\hline 3899.78 & $2295-27929$ & .35 & .080 & -1.10 & 4062.55 & $0-24608$ & .28 & .068 & -1.17 \\
\hline 3902.56 & $289-25906$ & .19 & .044 & -1.35 & 4066.80 & $8521-33104$ & .25 & .062 & -1.20 \\
\hline 3904.30 & $8347-33953$ & .73 & .17 & -.78 & 4067.76 & $6283-30860$ & .51 & .13 & -.90 \\
\hline 3904.56 & $5260-30863$ & .29 & .066 & -1.18 & 4071.11 & $12033-36589$ & 1.1 & .26 & -.58 \\
\hline 3904.85 & $8510-34112$ & .30 & .069 & -1.16 & 4074.49 & $1749-26285$ & .17 & .043 & -1.37 \\
\hline 3908.47 & $8394-33972$ & .29 & .067 & -1.17 & 4076.69 & $914-25437$ & .16 & .040 & -1.39 \\
\hline 3909.06 & $5790-31364$ & .20 & .045 & -1.35 & 4080.61 & $12033-36532$ & .90 & .23 & -.65 \\
\hline 3911.67 & $4420-29978$ & .31 & .072 & -1.14 & 4084.93 & $5791-30263$ & .24 & .059 & -1.23 \\
\hline 3914.20 & $12055-37596$ & .70 & .16 & -.79 & 4088.25 & $0-24453$ & .12 & .031 & -1.51 \\
\hline 3915.88 & $8423-33953$ & .64 & .15 & -.83 & 4090.14 & 1749-26191 & .66 & .17 & -.78 \\
\hline 3918.06 & $4421-29936$ & .12 & .028 & -1.56 & 4091.49 & $10198-34632$ & .51 & .13 & -.89 \\
\hline 3921.55 & $0-25492$ & .11 & .025 & -1.60 & 4094.62 & $11544-35960$ & .32 & .080 & -1.10 \\
\hline 3923.05 & $8394-33877$ & .29 & .067 & -1.17 & 4095.75 & $6283-30691$ & .28 & .070 & -1.15 \\
\hline 3924.27 & $4585-30060$ & .22 & .051 & -1.29 & 4098.03 & $289-24684$ & .18 & .046 & -1.34 \\
\hline
\end{tabular}


TABLE 2. Oscillator strengths for lines of U II-Continued

\begin{tabular}{|c|c|c|c|c|c|c|c|c|c|}
\hline $\begin{array}{c}\text { Wavelength } \\
\AA\end{array}$ & $\begin{array}{c}\text { Energy levels } \\
\mathrm{cm}^{-1}\end{array}$ & $\begin{array}{c}\text { gA } \\
10^{8 /} \mathrm{s} \\
\end{array}$ & gf & $\log g f$ & $\begin{array}{c}\text { Wavelength } \\
\AA\end{array}$ & $\begin{array}{c}\text { Energy levels } \\
\mathrm{cm}^{-1}\end{array}$ & $\begin{array}{c}\text { gA } \\
10^{8 /} \mathrm{s} \\
\end{array}$ & gf & $\log g f$ \\
\hline 4106.38 & $1749-26094$ & .20 & .050 & -1.30 & 4373.41 & $1749-24608$ & .073 & .021 & -1.68 \\
\hline 4106.93 & $0-24342$ & .098 & .025 & -1.60 & 4402.30 & $9075-31784$ & .077 & .022 & -1.65 \\
\hline 4113.11 & $0-24305$ & .065 & .017 & -1.78 & 4402.44 & $8510-31219$ & .11 & .033 & -1.48 \\
\hline 4116.10 & $0-24288$ & .25 & .064 & -1.19 & 4415.24 & $0-22642$ & .064 & .019 & -1.73 \\
\hline 4124.73 & $1749-25986$ & .21 & .053 & -1.28 & 4426.68 & $4706-27290$ & .082 & .024 & -1.62 \\
\hline 4128.34 & $4420-28636$ & .31 & .080 & -1.10 & 4427.65 & $289-22868$ & .056 & .017 & -1.78 \\
\hline 4133.20 & $5790-29978$ & .19 & .048 & -1.32 & 4433.89 & $8394-30941$ & .14 & .043 & -1.37 \\
\hline 4135.76 & $6283-30455$ & .15 & .039 & -1.40 & 4434.53 & $1749-24293$ & .070 & .021 & -1.68 \\
\hline 4136.81 & $4421-28587$ & .15 & .038 & -1.41 & 4462.97 & $915-23315$ & .075 & .022 & -1.65 \\
\hline 4138.66 & $9626-33781$ & .26 & .066 & -1.18 & 4465.13 & $2295-24684$ & .055 & .017 & -1.78 \\
\hline 4139.14 & $0-24152$ & .093 & .024 & -1.62 & 4472.34 & $289-22642$ & .17 & .052 & -1.28 \\
\hline 4141.23 & $8394-32535$ & .62 & .16 & -.80 & 4477.71 & $915-23241$ & .044 & .013 & -1.87 \\
\hline 4144.70 & $2295-26415$ & .11 & .029 & -1.54 & 4490.84 & $1749-24010$ & .068 & .021 & -1.69 \\
\hline 4145.39 & $8521-32638$ & .15 & .038 & -1.41 & 4510.32 & $0-22165$ & .038 & .012 & -1.94 \\
\hline 4155.41 & $6283-30341$ & .31 & .080 & -1.10 & 4515.28 & $289-22429$ & .091 & .028 & -1.56 \\
\hline 4163.68 & $0-24010$ & .14 & .036 & -1.44 & 4538.19 & $1749-23778$ & .096 & .030 & -1.53 \\
\hline 4164.79 & $289-24293$ & .049 & .013 & -1.90 & 4543.63 & $915-22917$ & .19 & .058 & -1.24 \\
\hline 4165.68 & $289-24288$ & .12 & .032 & -1.50 & 4545.58 & $2295-24288$ & .089 & .028 & -1.56 \\
\hline 4171.59 & $1749-25714$ & .46 & .12 & -.92 & 4553.86 & $915-22868$ & .029 & .0091 & -2.04 \\
\hline 4172.97 & $6283-30240$ & .20 & .051 & -1.29 & 4555.10 & $8394-30341$ & .15 & .046 & -1.34 \\
\hline 4174.19 & $5527-29476$ & .24 & .062 & -1.21 & 4567.69 & $1749-23635$ & .059 & .019 & -1.73 \\
\hline 4179.00 & $4585-28507$ & .15 & .039 & -1.41 & 4569.91 & $289-22165$ & .061 & .019 & -1.72 \\
\hline 4184.89 & $8521-32410$ & .19 & .049 & -1.31 & 4570.99 & $6283-28154$ & .066 & .021 & -1.68 \\
\hline 4188.07 & $289-24159$ & .094 & .025 & -1.61 & 4573.69 & $2295-24152$ & .091 & .029 & -1.54 \\
\hline 4189.28 & $289-24152$ & .13 & .034 & -1.47 & 4584.85 & $1749-23553$ & .041 & .013 & -1.89 \\
\hline 4197.52 & $8394-32211$ & .32 & .083 & -1.08 & 4601.13 & $915-22642$ & .035 & .011 & -1.95 \\
\hline 4200.10 & $6283-30085$ & .10 & .027 & -1.57 & 4603.66 & $2295-24010$ & .082 & .026 & -1.58 \\
\hline 4204.37 & $0-23778$ & .086 & .023 & -1.64 & 4605.15 & $5791-27499$ & .075 & .024 & -1.62 \\
\hline 4210.45 & $1749-25492$ & .065 & .017 & -1.76 & 4609.86 & $289-21975$ & .026 & .0082 & -2.09 \\
\hline 4211.66 & $4585-28322$ & .21 & .056 & -1.25 & 4622.43 & $5260-26887$ & .055 & .018 & -1.75 \\
\hline 4212.26 & $4421-28154$ & .14 & .038 & -1.42 & 4627.08 & $4585-26191$ & .17 & .054 & -1.27 \\
\hline 4214.42 & $289-24010$ & .052 & .014 & -1.86 & 4641.65 & $8394-29932$ & .12 & .038 & -1.42 \\
\hline 4227.33 & $6283-29932$ & .12 & .032 & -1.50 & 4646.60 & $915-22429$ & .088 & .029 & -1.54 \\
\hline 4228.76 & $4706-28347$ & .10 & .027 & -1.57 & 4666.86 & $289-21710$ & .058 & .019 & -1.72 \\
\hline 4232.04 & $289-23911$ & .047 & .013 & -1.90 & 4671.41 & $4585-25986$ & .090 & .030 & -1.53 \\
\hline 4240.59 & $5259-28834$ & .085 & .023 & -1.64 & 4689.07 & $0-21320$ & .063 & .021 & -1.68 \\
\hline 4241.67 & $4585-28154$ & .54 & .15 & -.83 & 4700.98 & $289-21555$ & .027 & .0088 & -2.05 \\
\hline 4244.37 & $0-23553$ & .17 & .046 & -1.33 & 4702.05 & $4706-25967$ & .057 & .019 & -1.73 \\
\hline 4252.43 & $4420-27930$ & .15 & .041 & -1.38 & 4702.52 & $2295-23553$ & .062 & .021 & -1.68 \\
\hline 4267.30 & $915-24342$ & .080 & .022 & -1.66 & 4722.73 & $1749-22917$ & .078 & .026 & -1.58 \\
\hline 4268.85 & $2294-25713$ & .068 & .019 & -1.73 & 4731.60 & $4585-25714$ & .099 & .033 & -1.48 \\
\hline 4269.61 & $1749--25163$ & .10 & .027 & -1.56 & 4755.73 & $0-21021$ & .043 & .014 & -1.84 \\
\hline 4273.98 & $915-24305$ & .055 & .015 & -1.82 & 4769.26 & 0-20961 & .035 & .012 & -1.92 \\
\hline 4276.47 & $5259-28636$ & .11 & .029 & -1.53 & 4772.70 & $2295-23241$ & .060 & .021 & -1.69 \\
\hline 4282.03 & $289-23635$ & .092 & .025 & -1.60 & 4779.63 & $914-21831$ & .030 & .010 & -1.98 \\
\hline 4282.46 & $4585-27929$ & .15 & .042 & -1.37 & 4819.54 & $4421-25163$ & .051 & .018 & -1.75 \\
\hline 4287.87 & $0-23315$ & .088 & .024 & -1.62 & 4847.65 & $2295-22917$ & .048 & .017 & -1.77 \\
\hline 4297.11 & $289-23553$ & .075 & .021 & -1.68 & 4858.08 & $4585-25163$ & .074 & .026 & -1.58 \\
\hline 4301.47 & $0-23241$ & .061 & .017 & -1.77 & 4859.68 & $0-20571$ & .033 & .012 & -1.93 \\
\hline 4310.39 & $6283-29476$ & .095 & .027 & -1.58 & 4861.02 & $5402-25967$ & .094 & .033 & -1.48 \\
\hline 4313.88 & $1749-24923$ & .080 & .022 & -1.65 & 4883.78 & $2294-22764$ & .033 & .012 & -1.92 \\
\hline 4319.78 & $2294-25437$ & .041 & .012 & -1.94 & 4886.33 & $5527-25986$ & .040 & .014 & -1.85 \\
\hline 4325.90 & $5526-28636$ & .099 & .028 & -1.56 & 4899.29 & $915-21320$ & .043 & .015 & -1.81 \\
\hline 4341.69 & $289-23315$ & .20 & .057 & -1.24 & 4913.16 & $2295-22642$ & .044 & .016 & -1.79 \\
\hline 4347.19 & $915-23911$ & .080 & .023 & -1.64 & 4924.64 & $5667-25967$ & .051 & .019 & -1.73 \\
\hline 4362.26 & $0-22917$ & .081 & .023 & -1.64 & 4933.66 & $4421-24684$ & .033 & .012 & -1.92 \\
\hline 4362.93 & $4585-27499$ & .084 & .024 & -1.62 & 4950.17 & $5791-25986$ & .040 & .015 & -1.83 \\
\hline 4372.57 & $915-23778$ & .082 & .024 & -1.63 & 4972.10 & $915-21021$ & .024 & .0089 & -2.05 \\
\hline
\end{tabular}


TABLE 2. Oscillator strengths for lines of U II-Continued

\begin{tabular}{|c|c|c|c|c|c|c|c|c|c|}
\hline $\begin{array}{c}\text { Wavelength } \\
\AA \\
\end{array}$ & $\begin{array}{c}\text { Energy levels } \\
\mathrm{cm}^{-1}\end{array}$ & $\begin{array}{c}\text { gA } \\
10^{8 /} \mathrm{s}\end{array}$ & gf & $\log g f$ & $\begin{array}{c}\text { Wavelength } \\
\AA \\
\end{array}$ & $\begin{array}{c}\text { Energy levels } \\
\mathrm{cm}^{-1}\end{array}$ & $\begin{array}{c}\text { gA } \\
10^{8 /} \mathrm{s}\end{array}$ & gf & $\log g f$ \\
\hline 4986.90 & $915-20961$ & .024 & .0089 & -2.05 & 5602.90 & $6445-24288$ & .031 & .014 & -1.84 \\
\hline 5008.22 & $1749-21710$ & .053 & .020 & -1.70 & 5603.97 & $5401-23241$ & .019 & .0088 & -2.06 \\
\hline 5047.41 & $2295-22101$ & .020 & .0076 & -2.12 & 5628.02 & $5791-23553$ & .020 & .0094 & -2.03 \\
\hline 5077.82 & $289-19977$ & .014 & .0055 & -2.26 & 5638.00 & $8394-26126$ & .029 & .014 & -1.85 \\
\hline 5085.86 & $915-20571$ & .021 & .0081 & -2.09 & 5644.21 & $12629-30341$ & .042 & .020 & -1.70 \\
\hline 5117.25 & 2294-21831 & .041 & .016 & -1.80 & 5653.77 & 2294-19977 & .016 & .0076 & -2.12 \\
\hline 5137.05 & $6445-25906$ & .031 & .012 & -1.92 & 5654.39 & $4421-22101$ & .022 & .011 & -1.97 \\
\hline 5145.08 & $6283-25714$ & .035 & .014 & -1.85 & 5664.86 & $5667-23315$ & .019 & .0092 & -2.04 \\
\hline 5154.04 & 2294-21691 & .022 & .0086 & -2.06 & 5674.88 & $9882-27499$ & .027 & .013 & -1.88 \\
\hline 5160.33 & $5791-25163$ & .075 & .030 & -1.52 & 5683.33 & $9553-27143$ & .041 & .020 & -1.70 \\
\hline 5184.59 & $5402-24684$ & .055 & .022 & -1.66 & 5691.40 & $6445-24010$ & .044 & .021 & -1.67 \\
\hline 5204.32 & $6283-25492$ & .054 & .022 & -1.66 & 5704.07 & $8379-25906$ & .040 & .020 & -1.71 \\
\hline 5225.12 & $5790-24923$ & .032 & .013 & -1.89 & 5706.99 & $9626-27143$ & .046 & .022 & -1.65 \\
\hline 5238.61 & $9553-28636$ & .048 & .020 & -1.71 & 5723.63 & $5401-22868$ & .037 & .018 & -1.74 \\
\hline 5247.35 & $5402-24453$ & .025 & .010 & -1.99 & 5733.21 & $8276-25714$ & .039 & .019 & -1.71 \\
\hline 5247.75 & $4585-23635$ & .041 & .017 & -1.77 & 5748.10 & $0-17392$ & .010 & .0051 & -2.30 \\
\hline 5257.04 & $5667-24684$ & .048 & .020 & -1.70 & 5748.44 & $8276-25667$ & .024 & .012 & -1.93 \\
\hline 5278.18 & $5402-24342$ & .029 & .012 & -1.91 & 5776.87 & $9626-26931$ & .029 & .015 & -1.84 \\
\hline 5288.40 & $5402-24305$ & .025 & .010 & -1.99 & 5788.59 & $6283-23553$ & .028 & .014 & -1.85 \\
\hline 5310.04 & 0-18827 & .012 & .0050 & -2.30 & 5791.74 & $9626-26887$ & .048 & .024 & -1.62 \\
\hline 5311.88 & $4421-23241$ & .036 & .015 & -1.82 & 5798.54 & $5401-22642$ & .052 & .026 & -1.58 \\
\hline 5312.73 & $5791-24608$ & .024 & .010 & -1.99 & 5811.27 & $8510-25714$ & .037 & .019 & -1.72 \\
\hline 5321.60 & $5667-24453$ & .023 & .010 & -2.00 & 5827.99 & $4706-21860$ & .019 & .0095 & -2.02 \\
\hline 5327.76 & $6283-25047$ & .044 & .019 & -1.73 & 5832.37 & $9882-27023$ & .041 & .021 & -1.68 \\
\hline 5349.92 & $4420-23107$ & .023 & .0099 & -2.01 & 5837.70 & $4585-21710$ & .039 & .020 & -1.70 \\
\hline 5362.40 & $9690-28333$ & .047 & .020 & -1.70 & 5839.04 & $7166-24288$ & .019 & .010 & -2.00 \\
\hline 5363.82 & $5667-24305$ & .025 & .011 & -1.97 & 5841.82 & $8379-25492$ & .033 & .017 & -1.78 \\
\hline 5368.43 & $9075-27698$ & .057 & .025 & -1.61 & 5843.29 & $6445-23553$ & .029 & .015 & -1.83 \\
\hline 5373.45 & $1749-20353$ & .0098 & .0042 & -2.37 & 5843.82 & $11787-28894$ & .040 & .020 & -1.69 \\
\hline 5386.19 & $15392-33953$ & .24 & .10 & -.99 & 5845.25 & $289-17392$ & .015 & .0075 & -2.12 \\
\hline 5403.20 & $5790-24293$ & .030 & .013 & -1.89 & 5853.91 & $1749-18827$ & .016 & .0081 & -2.09 \\
\hline 5405.98 & $8394-26887$ & .044 & .019 & -1.71 & 5859.16 & $13015-30078$ & .048 & .025 & -1.61 \\
\hline 5444.48 & $5791-24152$ & .039 & .017 & -1.76 & 5870.93 & $5402-22429$ & .032 & .017 & -1.78 \\
\hline 5465.68 & $9626-27917$ & .070 & .031 & -1.50 & 5886.93 & $8510-25492$ & .024 & .012 & -1.91 \\
\hline 5475.72 & $9241-27499$ & .11 & .052 & -1.29 & 5895.32 & $6283-23241$ & .028 & .014 & -1.84 \\
\hline 5480.27 & $12513-30756$ & .17 & .078 & -1.11 & 5932.44 & $5791-22642$ & .020 & .010 & -1.98 \\
\hline 5481.21 & $6445-24684$ & .068 & .031 & -1.51 & 5934.46 & $8510-25356$ & .022 & .012 & -1.94 \\
\hline 5482.54 & $15392-33627$ & .20 & .091 & -1.04 & 5952.05 & $6445-23241$ & .026 & .014 & -1.86 \\
\hline 5484.55 & 1749-19977 & .0094 & .0042 & -2.37 & 6004.83 & $9933-26581$ & .044 & .024 & -1.62 \\
\hline 5487.00 & $5791-24010$ & .038 & .017 & -1.76 & 6017.39 & $4706-21320$ & .024 & .013 & -1.89 \\
\hline 5491.22 & $8510-26716$ & .058 & .026 & -1.58 & 6051.74 & $914-17434$ & .021 & .011 & -1.94 \\
\hline 5492.97 & $0-18200$ & .044 & .020 & -1.70 & 6059.73 & $5667-22165$ & .020 & .011 & -1.95 \\
\hline 5494.66 & $4420-22615$ & .027 & .012 & -1.92 & 6067.23 & $914-17392$ & .018 & .010 & -2.00 \\
\hline 5501.49 & $9553-27725$ & .040 & .018 & -1.74 & 6087.34 & $6445-22868$ & .031 & .017 & -1.77 \\
\hline 5504.13 & $6445-24608$ & .046 & .021 & -1.68 & 6110.91 & $2294-18654$ & .0074 & .0042 & -2.38 \\
\hline 5527.83 & $12513-30599$ & .17 & .078 & -1.11 & 6164.77 & $10198-26415$ & .037 & .021 & -1.68 \\
\hline 5535.78 & $2294-20353$ & .013 & .0059 & -2.23 & 6181.37 & $8510-24684$ & .018 & .010 & -1.99 \\
\hline 5538.53 & $9075-27126$ & .047 & .022 & -1.66 & 6254.22 & $6445-22429$ & .020 & .012 & -1.93 \\
\hline 5544.81 & $4585-22615$ & .027 & .012 & -1.91 & 6279.64 & $5791-21710$ & .014 & .0083 & -2.08 \\
\hline 5548.05 & $12513-30533$ & .063 & .029 & -1.54 & 6280.20 & $5402-21320$ & .025 & .015 & -1.83 \\
\hline 5551.42 & $6445-24453$ & .036 & .017 & -1.78 & 6291.48 & $914-16804$ & .0049 & .0029 & -2.54 \\
\hline 5552.60 & $6283-24288$ & .026 & .012 & -1.92 & 6322.37 & $0-15812$ & .0067 & .0040 & -2.40 \\
\hline 5570.66 & $9553-27499$ & .073 & .034 & -1.47 & 6330.77 & $914-16706$ & .0057 & .0034 & -2.46 \\
\hline 5580.81 & $5402-23315$ & .025 & .012 & -1.94 & 6336.55 & $8510-24288$ & .016 & .0094 & -2.03 \\
\hline 5581.23 & $914-18827$ & .015 & .0070 & -2.15 & 6346.27 & $5401-21154$ & .0096 & .0058 & -2.24 \\
\hline 5581.61 & $289-18200$ & .019 & .0088 & -2.06 & 6374.78 & $10285-25967$ & .028 & .017 & -1.77 \\
\hline 5587.17 & $8521-26415$ & .026 & .012 & -1.92 & 6375.98 & $0-15679$ & .0037 & .0023 & -2.64 \\
\hline 5597.37 & $6445-24305$ & .035 & .017 & -1.78 & 6378.52 & $289-15962$ & .011 & .0067 & -2.17 \\
\hline
\end{tabular}


TABLE 2. Oscillator strengths for lines of U II-Continued

\begin{tabular}{|c|c|c|c|c|c|c|c|c|c|}
\hline $\begin{array}{c}\text { Wavelength } \\
\AA\end{array}$ & $\begin{array}{c}\text { Energy levels } \\
\mathrm{cm}^{-1}\end{array}$ & $\begin{array}{c}\mathbf{g A} \\
10^{8 /} \mathrm{s} \\
\end{array}$ & gf & $\log g f$ & $\begin{array}{c}\text { Wavelength } \\
\AA\end{array}$ & $\begin{array}{c}\text { Energy levels } \\
\mathrm{cm}^{-1}\end{array}$ & $\begin{array}{c}\text { gA } \\
10^{8 /} s\end{array}$ & gf & $\log g f$ \\
\hline 6379.64 & $5716-21387$ & .022 & .014 & -1.86 & 6776.89 & $10740-25492$ & .016 & .011 & -1.96 \\
\hline 6386.84 & $5667-21320$ & .013 & .0082 & -2.08 & 6796.43 & $6445-21154$ & .0058 & .0040 & -2.40 \\
\hline 6391.32 & $8510-24152$ & .014 & .0085 & -2.07 & 6808.76 & $8423-23106$ & .0078 & .0055 & -2.26 \\
\hline 6400.36 & $5402-21021$ & .010 & .0064 & -2.20 & 6876.75 & $8379-22917$ & .041 & .029 & -1.54 \\
\hline 6424.89 & $5402-20961$ & .013 & .0079 & -2.10 & 6948.58 & $16211-30599$ & .061 & .045 & -1.35 \\
\hline 6448.04 & $9933-25437$ & .015 & .0094 & -2.03 & 6987.72 & $10740-25047$ & .015 & .011 & -1.95 \\
\hline 6470.55 & $10740-26191$ & .017 & .011 & -1.97 & 7020.71 & $10444-24684$ & .029 & .022 & -1.66 \\
\hline 6485.38 & $6445-21860$ & .0087 & .0055 & -2.26 & 7073.61 & $4663-18797$ & .0059 & .0044 & -2.35 \\
\hline 6495.23 & $4585-19977$ & .0065 & .0041 & -2.38 & 7082.11 & $5401-19517$ & .011 & .0085 & -2.07 \\
\hline 6534.60 & $18654-33953$ & .057 & .036 & -1.44 & 7183.47 & $2294-16211$ & .0060 & .0046 & -2.33 \\
\hline 6535.46 & $914-16211$ & .0062 & .0040 & -2.40 & 7218.04 & $5667-19517$ & .0085 & .0066 & -2.18 \\
\hline 6536.58 & $5667-20961$ & .0096 & .0061 & -2.21 & 7379.63 & $10740-24288$ & .029 & .024 & -1.63 \\
\hline 6549.88 & $7166-22429$ & .012 & .0078 & -2.11 & 7454.03 & $10740-24.152$ & .024 & .020 & -1.69 \\
\hline 6557.58 & $10740-25986$ & .021 & .013 & -1.87 & 7510.08 & $8379-21691$ & .0098 & .0083 & -2.08 \\
\hline 6587.83 & $5526-20702$ & .0093 & .0060 & -2.22 & 7580.91 & $7166-20353$ & .010 & .0087 & -2.06 \\
\hline 6590.05 & $5402-20571$ & .0082 & .0053 & -2.27 & 7668.73 & $5790-18827$ & .0055 & .0049 & -2.31 \\
\hline 6603.34 & $2294-17434$ & .0032 & .0021 & -2.68 & 7669.69 & $9882-22917$ & .010 & .0092 & -2.04 \\
\hline 6621.77 & $2294-17392$ & .0098 & .0064 & -2.19 & 7802.40 & $10740-23553$ & .017 & .016 & -1.81 \\
\hline 6622.82 & $7547-22642$ & .013 & .0083 & -2.08 & 7837.71 & $9075-21831$ & .016 & .014 & -1.84 \\
\hline 6640.50 & $9553-24608$ & .0097 & .0064 & -2.19 & 7976.88 & $5667-18200$ & .0091 & .0086 & -2.06 \\
\hline 6671.21 & $8276-23262$ & .0079 & .0053 & -2.28 & 8040.10 & $8423-20857$ & .0065 & .0063 & -2.20 \\
\hline 6676.92 & $18654-33627$ & .082 & .055 & -1.26 & 8074.03 & $6445-18827$ & .010 & .0099 & -2.01 \\
\hline 6701.68 & $9690-24608$ & .012 & .0080 & -2.10 & 8188.20 & $644.5-18654$ & .010 & .010 & -2.00 \\
\hline 6707.59 & $5667-20571$ & .0074 & .0050 & -2.30 & 8210.27 & $10740-22917$ & .015 & .015 & -1.83 \\
\hline 6710.57 & 914- 15812 & .0035 & .0024 & -2.62 & 8307.56 & $1749-13783$ & .0028 & .0028 & -2.55 \\
\hline 6717.45 & $7547-22429$ & .014 & .0092 & -2.04 & 8337.50 & $5401-17392$ & .012 & .013 & -1.89 \\
\hline 6720.76 & $6445-21320$ & .0083 & .0056 & -2.25 & 8618.96 & 914-12513 & .0023 & .0026 & -2.58 \\
\hline 6726.89 & $8379-23241$ & .011 & .0076 & -2.12 & 8702.08 & $2294-13783$ & .0034 & .0038 & -2.42 \\
\hline 6742.47 & $5526-20353$ & .0051 & .0035 & -2.46 & 8787.37 & $4585-15962$ & .0065 & .0075 & -2.12 \\
\hline 6771.03 & $914-15679$ & .0030 & .0021 & -2.68 & & & & & \\
\hline
\end{tabular}

\section{References}

Bell, R. A. and Upson, W. L. (1971), Astrophys. Lett. 9, 109.

Corliss, C. H. and Bozman. W. R. (1962). Experimental transition probilities for spectral lines of seventy elements. Nat. Bur. $\mathrm{S}$ and. (U.S.), Monogr. 53, 583 pages.

Meggers, W. F., Corliss, C. H. and Scribner, B. F. (1975) Tables of spectral-line intensities. Nat. Bur. Stand. (U.S.) Monogr. 145. Part I, 403 pages.

Steinhaus, D. W., Phillips, M. V., Moody, J. B., Radziemski, L. J., Fisher, K. J., and Hahn, D. R., [1972] Los Alamos Report LA4977.

Voigt, P. A., [1975] Phys. Rev. A 11, 1845.

(Paper 80A3-895) 